\title{
Making neurorehabilitation fun: Multiplayer training via damping forces balancing differences in skill levels
}

\section{Conference Paper}

Author(s):

Baur, Kilian (1); Wolf, Peter (1); Riener, Robert; Duarte, Jaime E.

Publication date:

2017

Permanent link:

https://doi.org/10.3929/ethz-b-000252787

Rights / license:

In Copyright - Non-Commercial Use Permitted

Originally published in:

https://doi.org/10.1109/icorr.2017.8009359 


\title{
Making neurorehabilitation fun: Multiplayer training via damping forces balancing differences in skill levels
}

\author{
Kilian Baur ${ }^{1}$, Peter Wolf ${ }^{1}$, Robert Riener ${ }^{1}$, and Jaime E. Duarte ${ }^{1}$
}

\begin{abstract}
Multiplayer environments are thought to increase the training intensity in robot-aided rehabilitation therapy after stroke. We developed a haptic-based environment to investigate the dynamics of two-player training performing time-constrained reaching movements using the ARMin rehabilitation robot. We implemented a challenge level adaptation algorithm that controlled a virtual damping coefficient to reach a desired success rate. We tested the algorithm's effectiveness in regulating the success rate during game play in a simulation with computer-controlled players, in a feasibility study with six unimpaired players, and in a single session with one stroke patient. The algorithm demonstrated its capacity to adjust the damping coefficient to reach three levels of success rate (low [50\%], moderate [70\%], and high [90\%]) during singleplayer and multiplayer training. For the patient - tested in single-player mode at the moderate success rate only - the algorithm showed also promising behavior. Results of the feasibility study showed that to increase the player's willingness to play at a more challenging task condition, the effect of the challenge level adaptation - regardless of being played in single player or multiplayer mode - might be more important than the provision of multiplayer setting alone. Furthermore, the multiplayer setting tends to be a motivating and encouraging therapy component. Based on these results we will optimize and expand the multiplayer training platform and further investigate multiplayer settings in stroke therapy.
\end{abstract}

\section{INTRODUCTION}

The greatest benefits of therapy are seen in trainings with high level of intensity when patients train with high levels of mental and physical effort. To promote intensity during arm rehabilitation, researchers have developed multiplayer environments where two players can compete, or cooperate, with one another [1]. In these environments, a patient may interact with another patient, a therapist, a family member or a friend [2], [3]. This social context is thought to increase patients' motivation and levels of mental and physical effort. However, when more than one person is involved in training, for example during a competitive game, differences in skill level can affect the experience of success and their motivation to train.

To keep motivation high, the level of game difficulty can be adjusted to match the skill level. This approach has been previously discussed in the context of the model of

This work was supported by the Swiss National Science Foundation (Grant No. SNF-160313) through the National Centre of Competence in Research on Robotics (NCCR) and by the ETH Foundation through ETH Research Grant ETH-17 13-2.

${ }^{1}$ K. Baur, P. Wolf, R. Riener and J.E. Duarte are with the SensoryMotor Systems (SMS) Lab, Institute of Robotics and Intelligent Systems (IRIS), ETH Zurich, Switzerland and the Spinal Cord Injury Center, Balgrist University Hospital, University of Zurich, Switzerland kilian.baur@hest.ethz.ch flow [4]. Adjustments to the game's difficulty can include the core mechanics (e.g. the speed at which a ball travels in a game of tennis), the conditions in which a player performs the task (e.g. restricting the movement of a player), or a combination of the two. These adjustments can be made by a therapist supervising the training, by the players, or by algorithms embedded in the game. The final option is especially attractive in rehabilitation settings because it does not require additional input from users, can be performed systematically, and can target specific training goals [5], [6].

In these multiplayer environments, one aspect that requires special attention is how can these games be played by highly impaired individuals, i.e., those who could not perform the task withouth external assistance. Rehabilitation games played with passive devices already provide different modulators that can be adjusted to account for different skill levels of impaired players [7]. However, for this population, robotic devices offer unique design features that allow a given motor task to be tailored to the players' motor abilities [8]. In such setups, haptic force fields can be used to adjust the challenge level of a training task [9]. These force fields can be designed to support, or in some cases hinder, the player - thus changing the challenge level. In a multiplayer setting, this adaptation of the challenge level with haptic force fields can be applied individually for the different players.

The focus of our work is on the use of robotic devices to promote social interaction in rehabilitation training. We thus developed a multiplayer robot-based environment, where players of various skill levels can interact with each other using different input devices. This environment is based on a conceptual framework, derived from the flow model, where we seek to maintain high levels of motivation and engagement in training by balancing the differences in skill level between players [4]. Our focus is on time-constrained reaching movements because reaching is an essential component for functional upper-limb tasks alongside grasping, moving/manipulating and releasing [10]. The reaching movements are constrained in time by a defined time window when the given target position has to be reached.

We tested how an algorithm that adjusts the conditions of the task affects performance, effort and motivation during training. We first tested with simulated computer-players, then with a cohort of unimpaired participants, and finally with a single stroke patient. We hypothesized that by adjusting the damping level of the force field we could control a player's success rate. We further hypothesized that using this algorithm in a multiplayer setting would lead players to exhibit higher levels of motivation and effort. 


\section{METHODS, REQUIREMENTS AND IMPLEMENTATION}

\section{A. Framework}

The conceptual framework is largely derived from the flow model. This model relates the ideas of skill level and challenge level to predict the mental state of a person engaged in a given task [4]. When considering a multiplayer task, for example a two-player competitive game, the challenge level of the task is related to the skill level of the opponent. Since the skill level between players can vary greatly, so can the challenge level experienced by each player. In cases where the difference in skill level is large, the flow model predicts this and the game will result in sub-optimal mental states for the players. That, of course, is undesirable for rehabilitation training where we want patients to be highly motivated and engaged during training.

In our framework, we focus on the players' abilities to perform the task - measured as success rate - and define it as being opposed to the challenge level (i.e. higher success means lower challenge; Fig. 1). We then take a task condition - the damping coefficient $\mu$ in our current application - and adjust it to control the players' success rate. By controlling the success rate, we assume that we can balance the differences in skill levels of players. For training under balanced conditions, we predict the players to be closer to a flow-like state.

\section{B. Experimental setup}

1) Air hockey game: The air hockey game was set up similarly to a previous study [1]. The side walls constrained the movement of the puck, but not of the mallets. At the beginning of each match - and after each goal - the puck appeared in the center of the field. Both players then moved their mallets to the center of the field and a twosecond countdown began. During the countdown, an arrow indicating the start direction of the puck appeared. After the countdown, the puck moved in the direction indicated by the

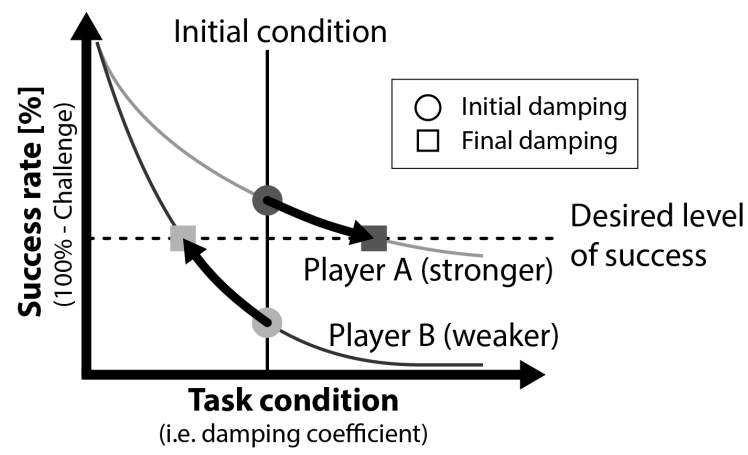

Fig. 1. Balancing the challenge level in robot assisted gaming. Two players start at the same initial task condition - i.e. damping coefficient - (vertical line). The stronger player $(\mathrm{A})$ (dark gray $\bigcirc$ ) achieves a higher success rate than the weaker player (B) (light gray $\bigcirc$ ). If we then adjust the task conditions separately for each player, we can increase the damping for player A and decrease it for player B until they achieve comparable success rates (dark gray $\square$ / light gray $\square$ ). If they now train under these task conditions we predict their mental states will be closer to a flow-like state during training. arrow and the match started. The score of both players was displayed on the right side of the playing field.

This version of the air hockey game can be played either in single-player mode or in multiplayer mode. The singleplayer mode features a computer opponent whose challenge level is adapted to the level of the player. The multiplayer mode features a human player using a computer mouse as the input device.

The air hockey game can be broken down into two main subtasks: defense and offense. In this study, we focused on the defense task. This task requires the player to move the mallet left and right in order to reach the approaching puck in time. Success in the defense task is to hit the puck forward; failure is the puck passing the mallet and reaching the player's goal zone. The success rate in this task is defined as the number of successful hits divided by the number of total trials.

2) Rehabilitation robot: In both single-player and multiplayer mode, the input device for the subject is the ARMin arm rehabilitation robot [11]. The elbow joint movement of the player was assessed by the position sensors in the robot and mapped to the player's mallet (Fig. 2).

We used a range of motion for the elbow between $30^{\circ}$ and $90^{\circ}$ and mapped from -12.3 [game distance units] to 12.3 [game distance units]. All other joints were initially fixed at a fully stretched pronated position: $90^{\circ}$ lateral shoulder abduction, $90^{\circ}$ horizontal shoulder adduction, $0^{\circ}$ upper arm rotation, $90^{\circ}$ lower arm pronation, $0^{\circ}$ wrist extension and closed hand module. To address the challenge level of the game, we virtually adjusted the damping coefficient at the elbow joint of the robot. The lower limit of the damping coefficient was set to $0 \mathrm{Nms} /{ }^{\circ}$. The upper limit of the damping was defined by the maximum torque that can be safely created by the motor and limited through voltage saturation at $59.5 \mathrm{Nm}$ in elbow flexion and extension.

3) Challenge level adaptation algorithm: The challenge level of the game can be modulated by parameters of the game core mechanics and the players' interface. However, changes in game core mechanics, such as puck speed, affect the difficulty level for both players generally. In contrast, changes at the player specific interface, such as damping

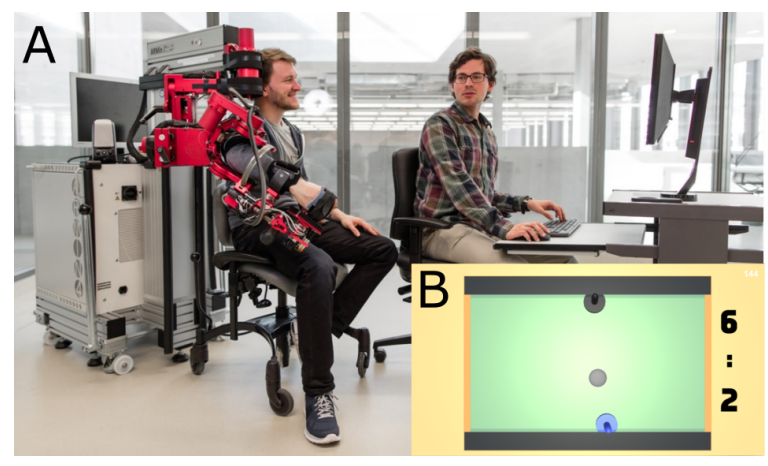

Fig. 2. Multiplayer setup. A: A subject uses the ARMin robot to play against a subject using the computer's mouse. The monitor displays the computer game to both players. B: The air hockey game environment. The puck moves on a field surrounded by four walls and two mallets defend two opposite goals. 
forces, affect the game for each player individually. We used an algorithm that adjusted the challenge level of the task by adapting the damping coefficient, based on the performance of the player [12]. This algorithm was previously tested in a single-player rehabilitation game where a timing component of the game was adapted in order to regulate the challenge level [13]. However, its applicability in a multiplayer setting has not yet been tested. The algorithm adapted the challenge level of the task by changing the damping coefficient $\mu$ as shown in Equation 1. The damping affects the maximum velocity that a player can reach and this, in turn, affects the ability of the player using the damped device to successfully defend his or her goal.

$$
\begin{array}{r}
\mu_{i}=\beta_{i} \cdot 0.01 \mathrm{Nms} /^{\circ}, \quad \beta_{1}=60 \\
\beta_{i+1} \begin{cases}\beta_{i}+\delta & \text { if success } \\
\beta_{i}-\alpha \delta & \text { if failure }\end{cases}
\end{array}
$$

Coefficients $\delta$ and $\alpha$ can be selected dependent on desired adaptation behavior. Coefficient $\delta$ defines the step size of the adaptation and coefficient $\alpha$ is defined by the desired success rates $p_{\infty}$ that is reached. The relation between $\alpha$ and $p_{\infty}$ is shown in Equation 3.

$$
\alpha=\frac{p_{\infty}}{1-p_{\infty}}
$$

\section{Artificial intelligence of the computer-player}

In our previous study [1], the movements of the computercontrolled mallet were governed by a P-type controller. We updated the movement behavior of the computer-controlled mallet to follow a human-like reaching behavior. We believe this would allow us to better compare the results between the single-player and multiplayer conditions. We assessed the reaching behavior of the participants in the ARMin robot in a series of pilot studies. Based on these assessments we modeled the computer's behavior using minimum jerk reaching movements [14]. We combined this minimum jerk model with normal distributions in reaction time $t_{r}$, endof-reach time $t_{e}$, and end-of-reach position $p_{e}$ to define the behavior of the computer opponent (Fig. 3).

\section{Study designs}

1) Multiplayer computer simulation: We tested the adaptive algorithm in a multiplayer setting by simulating humanlike players of different skill levels. In this simulation, the algorithm adapted the challenge level of the task by changing the coefficient of a virtual damping following Equation 1. We simulated three players of different skill levels by defining three maximum virtual forces, $F_{v, \text { max }}: 20$, 60 , and 100 virtual force units. These simulated players kept their maximum virtual forces constant throughout the game. The players competed against an opponent with an $F_{v, \text { max }}$ of 100 virtual force units whose damping coefficient remained constant. We expected stronger players to reach higher damping coefficients than weaker ones for the same success rate, which we set to $50 \%, 70 \%$, and $90 \%$. Coefficient $\delta$ was

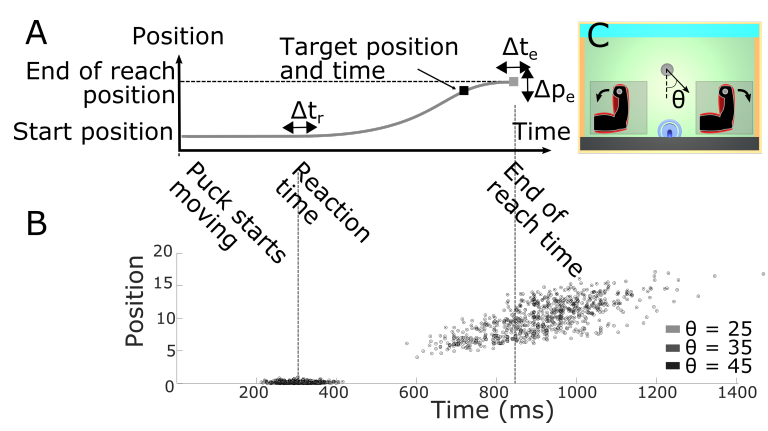

Fig. 3. Assessment based approach to model reaching behavior for computer-players. A: Schematic figure of assessed reaching movement of the players. After the puck starts moving the player starts moving with a delay of reaction time $t_{r}$. The puck can be reached within a restricted time with target position $p_{e}$ and target time $t_{e} . \mathrm{B}$ : The distributions for $\Delta t_{r}, \Delta p_{e}$ and $\Delta t_{e}$ were assessed with three different angles $\Theta=\left[25^{\circ}, 35^{\circ}, 45^{\circ}\right]$ in both flexion and extension direction. $\mathrm{C}$ : The puck and mallet movements were presented in the same game environment. The puck was always placed in the middle. Each trial started when the mallet was in the middle.

set to one for all simulations. Each simulated match lasted 16 minutes.

2) Feasibility study with healthy players: We tested the effect of the haptic field dynamically adapted to achieve specific success rates in a feasibility study with six healthy subjects (4 females; 25 to 31 years; right handed). Subjects were eligible if they met inclusion criteria (Table I). All participants played with the dominant arm. Subjects trained on two separate days. Both days started with an introduction phase of about four minutes. The subjects were given instructions and seated in the ARMin robot according to a standardized protocol [11]. They were then allowed to become familiar with the ARMin and the game environment during a test game. As part of the introduction, the subjects were instructed to focus on the defensive part of the game.

On the first day of testing, the game was played in either single-player or multiplayer mode (Fig. 4). On the second day, the game was played in the other mode. The order of modes was randomized. Each training mode was played in three different matches, each with a different desired success rate: low $=50 \%$, medium $=70 \%$, or high $=90 \%$. The order of the required success rate was randomized. Coefficient $\delta$ was set to one throughout all matches. Each match was played for eight minutes which was determined being a meaningful duration to play where the players did not express signs of physical fatigue. From each match, the temporal evolution of the damping coefficient was logged. The final damping coefficient provides a surrogate measure of the effort of patient in relation to his capability to apply forces.

TABLE I

INCLUSION CRITERIA

- Minimum age of 18 years

- Bodyweight under $120 \mathrm{~kg}$

- No serious medical or psychiatric disorder as reported by the participant

- No orthopaedic, rheumatic or other disease restricting movements of the paretic arm

- No shoulder subluxation of more than two fingers width

- No pacemaker or other implanted electronic device 
After each match, the subject rested for four minutes and responded to a motivation questionnaire. The motivation was measured with the Intrinsic Motivation Inventory (IMI) [15]. The IMI has previously been used with virtual environments for motor rehabilitation [8], [1]. We used four sub-scales of the IMI in this study: interest/enjoyment, effort/importance, pressure/tension, and perceived competence. For each subscale, we selected three statements including one inverse statement (Table II). Subjects rated how true each statement was on a 7-point scale, with 1 indicating "not at all true", and 7 indicating "very true".

3) Case study with one stroke patient: A 50-year-old female, four years after ischemic stroke in the left middle cerebral artery, and with moderate impairment of the right arm (Chedoke McMaster Stroke Assessment 3/7 for the arm and $4 / 7$ for the hand) agreed to participate and provided informed consent. She played the game in single-player mode during four minutes. We assessed the evolution of the damping coefficient during the match. This study as well as the study with the healthy players was approved by the responsible institutions (KEK-ZH-Nr. 2015-0013, Zurich, Switzerland, and clinicaltrials.gov NCT02720341).

\section{E. Data analysis}

To evaluate the performance of the algorithm - with both simulation and study data - we used a moving window. The size of this moving window was set to 50 trials in order to reduce noise in the success rate data. The moving window was used to calculate the success rate and the average damping coefficient experienced by the player during that window of trials. To evaluate the IMI data, the mean value of each sub-scale, for a given desired success rate in the multiplayer mode, was subtracted by the corresponding value in the single-player mode. The possible range for each score was therefore -6 to 6 .

\section{RESULTS}

\section{A. Computer simulation}

The algorithm adapted the damping coefficient for all three simulated players to reach close to the target success rates of

TABLE II

SELECTED STATEMENTS OF THE INTRINSIC MOTIVATION INVENTORY

Interest/enjoyment
- I enjoyed playing the air hockey game very much
- Playing the air hockey game was fun to do.
- Playing the air hockey game did not hold my attention at all.
(Inverse)
Effort/importance
- I put a lot of effort into this.
- I tried very hard on playing the air hockey game.
- I didn't put much energy into playing the air hockey game.
(Inverse)
Pressure/tension
- I felt very tense while playing the air hockey game
- I felt pressured while playing the air hockey game.
- I was very relaxed in playing the air hockey game. (Inverse)
Perceived competence
- I think I am pretty good at playing the air hockey game.
- I am satisfied with my performance at playing the air hockey
game.

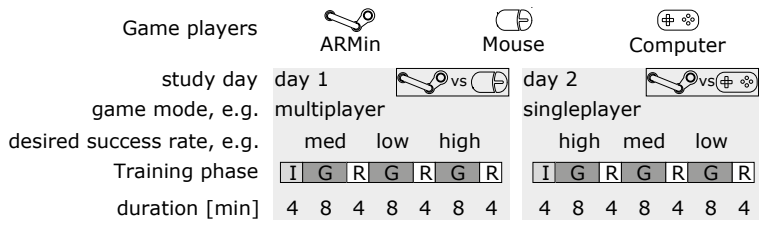

Fig. 4. Study protocol. The subjects played one day in single player mode and one day in multiplayer mode. On each day three different desired success rates for the subject were played: low $=50 \%$, medium $=70 \%$, high $=90$. The order of modes and the order of desired success rates were randomized. Three different actions were performed: The subjects were instructed (I, $4 \mathrm{~min}$ ), they played the game ( $\mathrm{G}, 8 \mathrm{~min})$ in defined mode and desired success rate, and they filled out the questionnaire while resting ( $R$, $4 \mathrm{~min}$ ) immediately after each play.

50\%, 70\% and 90\% (Fig. 5). All simulated players shifted away from the initial damping coefficients of $0.6 \mathrm{Nms} /{ }^{\circ}$ and $1.5 \mathrm{Nms} /{ }^{\circ}$ towards the the desired success rates. Stronger simulated players achieved higher damping coefficients than weaker players.

\section{B. Feasibility study}

For healthy players of different strengths, the damping coefficient was changed from the initial value of $0.60 \mathrm{Nms} /{ }^{\circ}$. All healthy players shifted away from their initial success rate towards the desired success rate. Exemplary data shows how the damping coefficient changes over time and how the players approach the desired success rate (Fig. 6A-C).

Four different agents were exposed to challenge level adaptation: Healthy player in ARMin playing against a computer-player, healthy player in ARMin playing against a human opponent playing by computer mouse, the computerplayer playing against a healthy player in ARMin, and a patient in ARMin playing against a computer-player (Fig. 6D).

\section{Effects on motivation}

The difference of multiplayer and single player mode in final damping coefficient, for the total IMI score and for all sub-scales was tested (Fig. 7). A one sided paired-samples t-test was conducted for the final damping coefficient, total IMI score and all sub-scales to compare the differences of multiplayer and single player to no difference after correcting for multiple tests $\left(\mathrm{p}^{*}=0.05 / 15\right)$ (Table III).

\section{DISCUSSION}

We implemented a novel type of dynamic challenge level adaptation using haptics as the modulator of challenge level. We tested this adaptation of challenge level in a robot-based environment in single- and multiplayer setups. The algorithm adjusted the forces experienced by all players - simulated, unimpaired, and impaired - to achieve the desired levels of success rate experienced during training.

The majority of the human subjects in the study approached the desired success rates. This result was not affected by the challenge-adapted computer-player nor by the multiplayer dynamics of playing against the human opponent playing with the mouse. One particularly strong human 
A

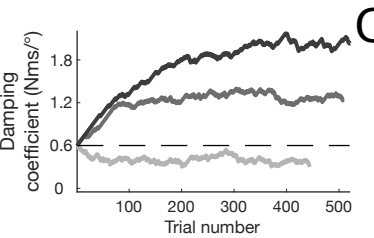

B

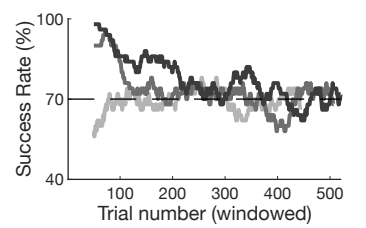

D

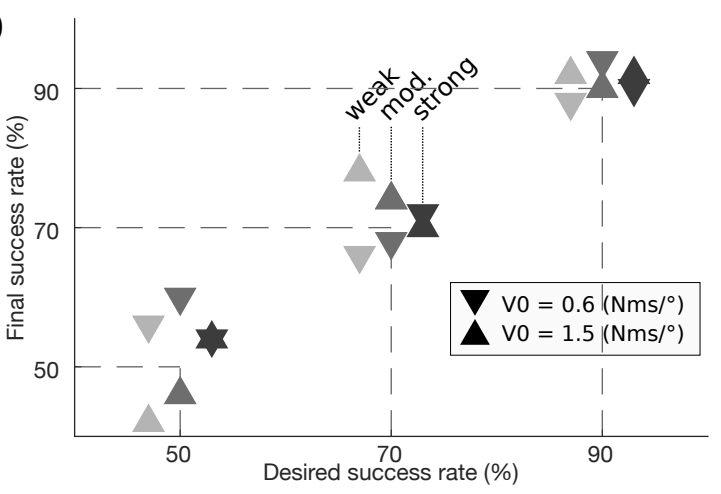

Fig. 5. Dynamics of the challenge level adaptation in simulations. A-C: Exemplifying evolution of three simulated players' data presented as (A) the damping coefficient over trial number, (B) the success rate over windowed trial number and $(\mathrm{C})$ the success rate over the damping coefficient: The simulated players were modeled as a strong player (dark gray), moderate player (medium gray) and weak player (light gray) with strength levels of 20, 60 and 100 virtual force units, respectively. All started with a damping coefficient of $0.6 \mathrm{Nms} /{ }^{\circ}$. The damping coefficient and the success rate evolve from that initial state to a final state targeting a desired success rate of $70 \%$. The start point $(\bigcirc)$ indicates the damping coefficient and success rate after the first windowed trial number. The end point $(\square)$ indicates the final damping coefficient and the success rate of the last windowed trial number. We considered a window size of 50 trials. D: Final success rate for the simulated matches: The players were simulated using two different initial damping coefficients of $0.6 \mathrm{Nms} /{ }^{\circ}(\nabla)$ and $1.5 \mathrm{Nms} /{ }^{\circ}(\triangle)$ targeting three different desired success rates of $50 \%, 70 \%$ and $90 \%$, respectively.

subject reached the force limits of the robotic device when targeting the moderate and low success rates. The damping could not increase more since the motor torques were limited due to safety reasons. This can be solved by the use of an additional challenge level modulator related to the interface device, e.g., change in joint angle mapping, being applied whenever reaching the limits of the robot.

In the other extreme, at the desired high success rate, some subjects reached a ceiling in their success rate when approaching damping coefficients close to $0 \mathrm{Nms} /{ }^{\circ}$. This could be the result of the lower viscosity forcing the player to control for higher accelerations of the reaching movements. Beyond the dynamics of the robot, another explanation could be that the subjects had to adapt to changes in damping that were better perceived at lower damping coefficients due to the fix step size $\delta$ that causes changes of a higher factor at this damping level. In particular, in the case of failure at high desired success rate the factor of change can be

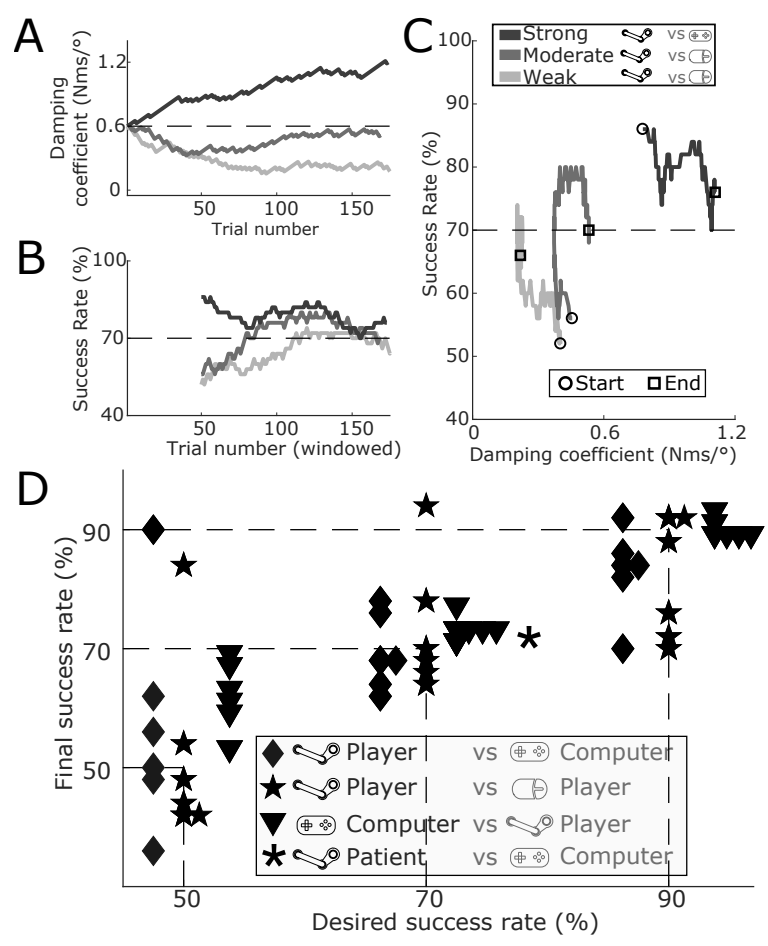

Fig. 6. Dynamics of the challenge level adaptation in human players. A-C: Exemplifying evolution of three healthy players' data presented as (A) the damping coefficient over trial number, (B) the damping coefficient over windowed trial number and $(C)$ the success rate over the damping coefficient: The presented evolution shows a moderate and a stronger player (identified by results) playing in single-player mode (medium gray and dark gray) and a weaker player playing in multiplayer mode (light gray). All started with a damping coefficient of $0.6 \mathrm{Nms} /{ }^{\circ}$. The damping coefficient and the success rate evolve from that initial state to a final state targeting a desired success rate of $70 \%$. The start point $(\bigcirc)$ indicates the damping coefficient and success rate after the first windowed trial number. The end point $(\square)$ indicates the final damping coefficient and the success rate of the last windowed trial number. We considered a window size of 50 trials. D: Final success rate for all matches of all human players and computer-players: The players started with an initial damping coefficient of $0.6 \mathrm{Nms} /{ }^{\circ}$. The human players playing against a computer-player $(\diamond)$, the human players playing against another human player playing with mouse $(\star)$, and the computer-players playing against a human player $(\nabla)$ played matches targeting different desired success rates of $50 \%, 70 \%$ and $90 \%$, respectively. The patient $(*)$ was targeting a desired success rate of $70 \%$.

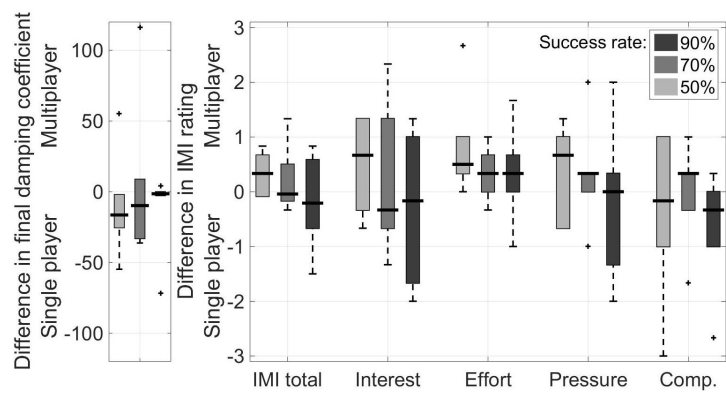

Fig. 7. Difference in final damping coefficient and IMI scores of multiplayer and single-player mode. For each desired success rate $(50 \%, 70 \%$ and $90 \%)$ the final damping coefficient and the mean scores of the total IMI and the IMI sub-scales are presented. No significant difference in the final damping coefficient, in the mean scores of the total IMI and in all sub-scales (interest/enjoyment, effort/importance, pressure/tension, perceived competence) can be observed after correcting for multiple tests $\left(\mathrm{p}^{*}=0.05 / 15\right)$. 
TABLE III

P-VALUES OF MULTIPLAYER AND SINGLE-PLAYER COMPARISON

\begin{tabular}{|r||c|c|c|}
\hline Desired success rate & $50 \%$ & $70 \%$ & $90 \%$ \\
\hline \hline Final damping coefficient; $\mathrm{p}=$ & 0.73 & 0.40 & 0.82 \\
Final Total IMI; $\mathrm{p}=$ & 0.05 & 0.22 & 0.69 \\
\hline interest/enjoyment; $\mathrm{p}=$ & 0.11 & 0.39 & 0.68 \\
effort/importance; $\mathrm{p}=$ & 0.04 & 0.07 & 0.20 \\
pressure/tension; $\mathrm{p}=$ & 0.16 & 0.22 & 0.61 \\
perceived competence; $\mathrm{p}=$ & 0.72 & 0.50 & 0.91 \\
\hline
\end{tabular}

high (e.g. a change in damping coefficient of $0.09 \mathrm{Nms} /{ }^{\circ}$, from $0.11 \mathrm{Nms} /{ }^{\circ}$ to $0.02 \mathrm{Nms} /{ }^{\circ}$. Thus, to achieve higher success rates - and especially for patients whose inherent skill level may be lower - we suggest to migrate from the damping based challenge level modulation towards a supportive control challenge level modulation. Such supportive control can be based on an adaptive impedance controller along a minimum jerk trajectory as it implemented for the computer-player already.

By visual inspection, a steady state at the desired success rate was not achieved for low and high desired success rate due to the small step size of the algorithm. This progress can be boosted by setting the initial challenge level based on the desired success rate and the force of the subject (e.g. based on a pre-evaluation of force). The step size $\delta$ could also be dynamically adapted to be higher at the start of the game and then decreased during play time, to enable fast adaptation at the beginning.

There were no significant differences in the final damping coefficient reached when comparing multiplayer to singleplayer mode. However, the results show the tendency that for low desired success rates subjects approached a higher final damping coefficient in the single-player mode. That is contradictory to our hypothesis whereby we expected that higher motivation from social interaction would lead to higher physical effort exerted during training. Considering the limitation of our study that in the multiplayer game the challenge level was only adapted for the subject but not for the opponent, this emphasizes the importance of adapting the challenge level on both players in a multiplayer game. A further limitation is the assumption that the task can be reduced to a defensive task.

Regarding the subjective reports of motivation, effort, and competence (IMI questionnaire), we did not see significant differences between the single-player and multiplayer modes. Considering the total IMI-score, however, there is a tendency that the multiplayer mode can be more motivating, especially at lower desired success rates, than the single-player mode.

\section{CONCLUSIONS}

Multiplayer games in rehabilitation have potential to boost effort and intrinsic motivation for patients. To fully employ their potential it is necessary to balance the different skill levels of players. Damping forces can modulate the challenge level for differently skilled players. In the presented feasibility study, the effort of a player could be optimized by adjusting the damping coefficient based on current success rate.
This feasibility study in a multiplayer setting supports the generalizability of the algorithm beyond single-player application towards multiplayer games using a robotic exoskeleton as an input device. Based on these promising results we will continue to test the algorithm on impaired participants and further investigate its impact on motivation in a multiplayer setting in combination with difficulty adaptation for both human players.

\section{ACKNOWLEDGMENT}

The authors would like to thank the participants, patients, and therapists involved in the training.

\section{REFERENCES}

[1] D. Novak, A. Nagle, U. Keller, and R. Riener, "Increasing motivation in robot-aided arm rehabilitation with competitive and cooperative gameplay." Journal of neuroengineering and rehabilitation, vol. 11, no. 1, p. 64, 2014.

[2] M. Goršič, I. Cikajlo, and D. Novak, "Competitive and cooperative arm rehabilitation games played by a patient and unimpaired person: effects on motivation and exercise intensity," Journal of NeuroEngineering and Rehabilitation, vol. 14, no. 1, p. 23, 2017.

[3] G. Ganesh, A. Takagi, R. Osu, T. Yoshioka, M. Kawato, and E. Burdet, "Two is better than one: physical interactions improve motor performance in humans." Scientific reports, vol. 4, p. 3824, jan 2014.

[4] M. Csikszentmihalyi, Flow and the foundations of positive psychology. Springer, 2014.

[5] M. Maier, B. R. Ballester, E. Duarte, A. Duff, and P. F. M. J. Verschure, "Social Integration of Stroke Patients through the Multiplayer Rehabilitation Gaming System," pp. 100-114, 2014.

[6] G. a. P. Caurin, A. a. G. Siqueira, K. O. Andrade, R. C. Joaquim, and H. I. Krebs, "Adaptive strategy for multi-user robotic rehabilitation games." Conference proceedings : ... Annual International Conference of the IEEE Engineering in Medicine and Biology Society. IEEE Engineering in Medicine and Biology Society. Conference, vol. 2011, no. 1, pp. 1395-8, jan 2011.

[7] M. Goršič, A. Darzi, and D. Novak, "Comparison of two difficulty adaptation strategies for competitive arm rehabilitation exercises," IEEE International Conference on Rehabilitation Robotics, vol. 2017 July, pp. 1-6, 2017.

[8] R. Colombo, F. Pisano, A. Mazzone, C. Delconte, S. Micera, M. C. Carrozza, P. Dario, and G. Minuco, "Design strategies to improve patient motivation during robot-aided rehabilitation." Journal of neuroengineering and rehabilitation, vol. 4, p. 3, jan 2007.

[9] L. Marchal-Crespo and D. J. Reinkensmeyer, "Review of control strategies for robotic movement training after neurologic injury." Journal of neuroengineering and rehabilitation, vol. 6, p. 20, jan 2009.

[10] C. Lang, M. Strube, M. Bland, K. Waddell, K. Cherry-Allen, R. Nudo, A. Dromerick, and R. Birkenmeier, "Dose-response of task-specific upper limb training in people at least 6 months post stroke: A Phase II, single-blind, randomized, controlled trial." Annals of Neurology, pp. 1-13, 2016.

[11] M. Guidali, A. Duschau-Wicke, S. Broggi, V. Klamroth-Marganska, T. Nef, and R. Riener, "A robotic system to train activities of daily living in a virtual environment," Med. Biol. Eng. Comput., vol. 49, no. 10, pp. 1213-1223, 2011.

[12] S. Spencer, "Movement training and post-stroke rehabilitation using a six degree of freedom upper-extremity robotic orthosis and virtual environment," Ph.D. dissertation, University of California, Irvine, 2009.

[13] H. Taheri, J. B. Rowe, D. Gardner, V. Chan, K. Gray, C. Bower, D. J. Reinkensmeyer, and E. T. Wolbrecht, "Design and preliminary evaluation of the finger rehabilitation robot: controlling challenge and quantifying finger individuation during musical computer game play," J. Neuroeng. Rehabil, vol. 11, no. 1, p. 10, 2014.

[14] T. Flash and N. Hogan, "The coordination of arm movements: an experimentally confirmed mathematical model." The Journal of neuroscience, vol. 5, no. 7, pp. 1688-1703, 1985.

[15] E. McAuley, T. Duncan, and V. V. Tammen, "Psychometric properties of the Intrinsic Motivation Inventory in a competitive sport setting: a confirmatory factor analysis." pp. 48-58, 1989. 